\title{
Peritubular Capillary Oxygen Consumption in Sepsis-Induced AKI: Multi-Parametric Photoacoustic Microscopy
}

\author{
Nabin Poudel ${ }^{a}$ Shuqiu Zheng ${ }^{a}$ Colleen M. Schinderle ${ }^{a}$ Naidi Sun ${ }^{b}$ Song Hu ${ }^{b}$ \\ Mark D. Okusa ${ }^{a}$ \\ ${ }^{a}$ Division of Nephrology and Center for Immunity, Inflammation and Regenerative Medicine, University of Virginia, \\ Charlottesville, VA, USA; ' Department of Biomedical Engineering, Washington University at St. Louis, St. Louis, MO, USA
}

\section{Keywords}

Acute kidney injury · Sepsis · Hemodynamics · Oxygen

metabolism · Photoacoustic microscopy

\begin{abstract}
Understanding and measuring parameters responsible for the pathogenesis of sepsis-induced AKI (SI-AKI) is critical in developing therapies. Blood flow to the kidney is heterogeneous, partly due to the existence of dynamic networks of capillaries in various regions, responding differentially to oxygen demand in cortex versus medulla. High energy demand regions, especially the outer medulla, are susceptible to hypoxia and subject to damage during SI-AKI. Proximal tubule epithelial cells in the cortex and the outer medulla can also undergo metabolic reprogramming during SI-AKI to maintain basal physiological status and to avoid potential damage. Current data on the assessment of renal hemodynamics and oxygen metabolism during sepsis is limited. Preclinical and clinical studies show changes in renal hemodynamics associated with SI-AKI, and in clinical settings, interventions to manage renal hemodynamics seem to help improve disease outcomes in some cases. Lack of proper tools to assess temporospatial changes in peritubular blood flow and tissue oxygen metabolism is a barrier to our ability to understand microcirculatory dynamics and oxygen consumption and their role in the pathogenesis of SI-AKI. Cur-
\end{abstract}

rent tools to assess renal oxygenation are limited in their usability as these cannot perform continuous simultaneous measurement of renal hemodynamics and oxygen metabolism. Multi-parametric photo-acoustic microscopy (PAM) is a new tool that can measure real-time changes in microhemodynamics and oxygen metabolism. Use of multi-parametric PAM in combination with advanced intravital imaging techniques has the potential to understand the contribution of microhemodynamic and tissue oxygenation alterations to SI-AKI.

(c) 2020 S. Karger AG, Basel

\section{Pathophysiology of Sepsis-Induced Acute Kidney Injury}

Sepsis is described as organ dysfunction resulting from aberrant host response to an infection [1]. Acute kidney injury (AKI) develops in nearly $65 \%$ of patient with sepsis and further complicates the therapeutic management of sepsis [2]. Moreover, patients with AKI can also develop

Contribution from the AKI and CRRT 2020 Symposium at the twentyfifth International Conference on Advances in Critical Care Nephrology, Manchester Grand Hyatt, San Diego, CA, USA, February 24-27, 2020. This symposium was supported in part by the NIDDK funded University of Alabama at Birmingham-University of California San Diego O'Brien Center for Acute Kidney Injury Research (P30DK079337).

$\begin{aligned} & \text { karger@karger.com } \\ & \text { www.karger.com/nef }\end{aligned}$
Karger $\stackrel{\text { ' } 2020 \text { S. Karger AG, Basel }}{ }$


sepsis as a consequence [3]. Pathophysiology of SI-AKI is complex and begins with activation of innate immune system mediated by binding of pathogen-associated molecular pattern (e.g., bacterial endotoxins) or damage-associated molecules released by host cells (e.g., extracellular ATP, mitochondrial DNA, and other metabolites) to their receptors present in the host cell surface and subsequent release of inflammatory mediators [4]. This evoked immune response then leads to a wide array of pathophysiological changes including dysregulated homeostasis, immunosuppression, cellular, tissue, and organ dysfunction [5]. Sepsis-mediated microcirculatory dysfunction primarily occurs by direct damage to the glycocalyx lining the endothelium of microvessels or by inflammation-induced aberrant vasoreactivity due to release of vasoactive substances by the endothelium, tubule epithelial cells, and vascular smooth muscle cells, leading to tissue hypoperfusion, reduced oxygen delivery, and organ dysfunction [6]. Moreover, endothelial activation due to inflammation further leads to leukocyte adhesion and rolling, capillary leakage, interstitial edema, leukostasis, and tissue hypoperfusion. In the kidney, sepsis-mediated microcirculatory dysfunction results in local ischemia, tubular dysfunction, and injury [7]. At cellular level, mitochondrial dysfunction and reactive oxygen species (ROS) production during sepsis contribute to tubular cell death $[8,9]$.

\section{Renal Oxygen Delivery in Normal Health}

Three distinct types of vascular beds with different flow dynamics are important in the delivery of oxygen and other nutrients to the kidney. These include glomerular capillaries, cortical peritubular capillaries, and medullary vasa recta. $\mathrm{RBF}$ in the medulla is significantly lower than the cortex. RBF in the medulla contributes only $10 \%$ of the total kidney RBF [10]. Furthermore, different regions of the medulla are also heterogeneously perfused. Compared to the cortex, RBF in the outer medulla is about $40 \%$ while the inner medulla is only $10 \%$ [11]. Kidneys account for $20 \%$ of total oxygen consumption. The kidney cortex has a tissue oxygen tension $\left(\mathrm{PO}_{2}\right)$ of $\sim 50 \mathrm{~mm} \mathrm{Hg}$, and that of the medulla is only about $10-20 \mathrm{~mm} \mathrm{Hg}$ [12]. This is primarily due to differential oxygen demand, dependent on tubular sodium reabsorption [13]. Mathematical models also suggest that luminal flow in the proximal tubule is an important determinant of tissue $\mathrm{PO}_{2}$ in the cortex [14].

Tubule epithelial cells from S3 segment of the proximal tubule in the corticomedullary junction perform ATP-dependent active transport and utilize oxygen-dependent ox- idative phosphorylation (OXPHOS) and beta-oxidation of fatty acids (FAO) for energy production, while other tubule components in the inner medulla rely on glycolysis for energy production [15]. Because of this disparity of blood flow, oxygen tension, and energy source in cortex and medulla, the kidney is susceptible to injury. However, there exists a strong renal adaptive mechanism to maintain constant $\mathrm{PO}_{2}$ and normal tubular function and prevent cellular damage during altered oxygen supply [16]. At the tissue level, an increase in RBF or oxygen delivery is largely compensated by elevated oxygen consumption and partly by arteriovenous shunting [17]. At the cellular level, hypoxia and hypoxia-inducible factor signaling induce renal peritubular fibroblasts to increase synthesis erythropoietin and increase oxygen-carrying capacity [16].

\section{Renal Oxygen Delivery in SI-AKI}

Sepsis results in microcirculation dysfunction [18]. Preclinical and clinical studies show divergent renal hemodynamics during SI-AKI. Sepsis associated increase in cardiac output can potentially increase or preserve RBF [19]. In humans with sepsis, RBF declines [20,21]. Though Prowle et al. [20] did not find an association between reduced RBF and renal function in septic patients, the early decline in RBF and oxygen metabolism were associated with increased tubular injury in the study by Skytte Larsson et al. [21]. However, Tran et al. [22] using blood oxygen level-dependent (BOLD) MRI did not observe overt changes in renal tissue oxygenation despite reduced oxygen delivery in mice during LPS-induced AKI. Wang et al. [23], using pimonidazole-protein adducts to assess renal tubular hypoxia, observed cortical hypoxia in a caecal ligation puncture model as early as $4 \mathrm{~h}$ after surgery. The inconsistencies among these findings could largely be due to the complex pathogenesis of sepsis, as well as due to the variation in experimental approaches to assess renal oxygenation in different studies.

\section{Metabolic Reprogramming in the Kidney during SI-AKI}

Another key event that contributes to SI-AKI is metabolic reprogramming. It was first identified in rapidly dividing cancer cells where a lack of oxygen supply switched their metabolism from aerobic respiration to anaerobic respiration, a phenomenon known as Warburg effect [24]. Warburg effect has been observed in many other cell types beside cancer cells. During sepsis, the metabolic re- 
Table 1. Applicability and limitation of current techniques to assess renal hemodynamics and oxygenation

\begin{tabular}{|c|c|c|c|c|c|c|c|}
\hline Method & Technique & Primary parameter of measurement & \multicolumn{4}{|c|}{ Measurable parameters* } & $\begin{array}{l}\text { Ability to } \\
\text { perform } \\
\text { repetitive } \\
\text { measurement }\end{array}$ \\
\hline PIM & IHC & Extent of hypoxia & $\mathrm{N}$ & $\mathrm{Y}$ & $\mathrm{N}$ & $\mathrm{N}$ & $\mathrm{N}$ \\
\hline Oxygen electrodes & Oxygen probes & $\mathrm{O}_{2}$ concentration & $\mathrm{N}$ & $\mathrm{Y}$ & $\mathrm{Y}$ & $\mathrm{N}$ & $\mathrm{Y} / \mathrm{N}$ \\
\hline MRI & DNP-MRI & Resonance imaging of $\mathrm{O}_{2}$ sensitive magnetic agent & $\mathrm{N}$ & $\mathrm{Y}$ & $\mathrm{Y}$ & $\mathrm{N}$ & Y \\
\hline \multirow{2}{*}{$\begin{array}{l}\text { Microscopy } \\
\text { imaging }\end{array}$} & PLIM & Phosphorescence quenching property of $\mathrm{O}_{2}$ & Y & $\mathrm{Y}$ & $\mathrm{Y}$ & $\mathrm{N}$ & $\mathrm{Y}$ \\
\hline & PAM & Absorption spectrum of oxy- and deoxy-hemoglobin & $\mathrm{Y}$ & $\mathrm{Y}$ & $\mathrm{Y}$ & $\mathrm{Y}$ & $\mathrm{Y}$ \\
\hline
\end{tabular}

IHC, immunohistochemistry; BOLD-MRI, blood oxygen level-dependent MRI; DNP-MRI, dynamic nuclear polarization MRI; PLIM, phosphorescence lifetime imaging microscopy; PAM: photoacoustic microscopy; $\mathrm{Y}$, yes; $\mathrm{N}$, no. * Only parameters relevant to the review are mentioned here. ${ }^{\#} \mathrm{BOLD}$-MRI measures blood oxygenation.

programming is biphasic, initial glucose-dependent anabolic phase followed by OXPHOS and FAO dependent catabolic phase [25]. Activation of the innate immune system and reduced oxygen delivery to the proximal tubule cells induces HIFla signaling and switches cells toward less efficient anaerobic glycolysis for ATP production [26] followed by activation of the adenosine monophosphate kinase pathway [27]. This switch to anaerobic glycolysis could serve multiple purposes: (1) by undergoing less efficient pathway of energy production, cells can still maintain ATP production to maintain basal cellular activity and avoid potential cell death [24], (2) reduced OXPHOS activity leads to less mitochondrial ROS production and less cellular damage [28], and (3) activation of pentose phosphate pathway leads to increased production of nicotinamide adenine dinucleotide $(\mathrm{NAD}+)$ [29], a potent ROS inhibitor that exerts protection during SIAKI [9]. Paradoxically, the metabolic switch could also contribute to AKI pathology. It has been shown that improving OXPHOS early on during SI-AKI in mice results in improved outcome [30], so do the late activation of AMPK and Sirtuin-1 [25]. Understanding these temporal changes in metabolism during SI-AKI could serve as the key to identifying metabolic targets.

\section{Multi-Parametric Photo-Acoustic Microscopy}

Current techniques available for assessment of renal oxygenation employ either oxygen microelectrodes [31] (polarographic electrodes), optical probes (optodes),
MRI techniques including blood oxygenation level-dependent MRI (BOLD-MRI) [22] and dynamic nuclear polarization MRI (DNP-MRI) with an oxygen-sensitive paramagnetic agent (OX63) [32], and phosphorescence lifetime imaging microscopy using phosphorescence tracer element [33] (Table 1). While classical oxygen microelectrode and optode-based techniques can directly measure renal oxygenation in the precise location in the kidneys, these techniques cannot give a continuous measurement. MRI-based techniques can provide continuous assessment of renal oxygenation but accurate quantification of the oxygenation is still a hindrance. Phosphorescence lifetime imaging microscopy can provide the status of hypoxia at molecular details, but the low penetration of intravital imaging allows measurement of changes only in the cortex. Besides, none of the currently existing techniques can provide simultaneous measurement of blood flow, oxygen saturation, and oxygen extraction.

Multi-parametric PAM is a novel technique that allows intravital high-resolution, quantitative, and comprehensive characterization of hemodynamics and oxygen metabolism and associated microvascular pathology in animal models [34]. PAM enables concurrent imaging of blood perfusion, oxygenation, and flow without the use of any tracers of fluorophore molecules (Fig. 1). These parameters can then be used to derive other parameters including oxygen extraction fraction and metabolic rate of oxygen. By distinguishing the spectra of oxy- and deoxy-hemoglobin, repetitive pulsed dual-wavelength excitation provides details on oxygen concentration and satu- 
Fig. 1. Photoacoutic microscopy. Photoacoustic microscopy is a hybrid of optics and ultrasound. In photoacoustic microscopy, usually, a focused laser pulse is directed into biological tissue (kidney). The tissue absorbs light and induces transient heating. The thermoelastic expansion of the tissue converts the heat into acoustic emission. A transducer outside of the tissue can detect the acoustic wave and form an image. The conversion from optical absorption to acoustic emission has a few unique advantages. First, photoacoustic imaging is solely sensitive to optical absorption creating a very specific imaging contrast. Second, relying on focused acoustic detection photoacoustic microscopy can operate beyond the penetration of pure optical microscopy.

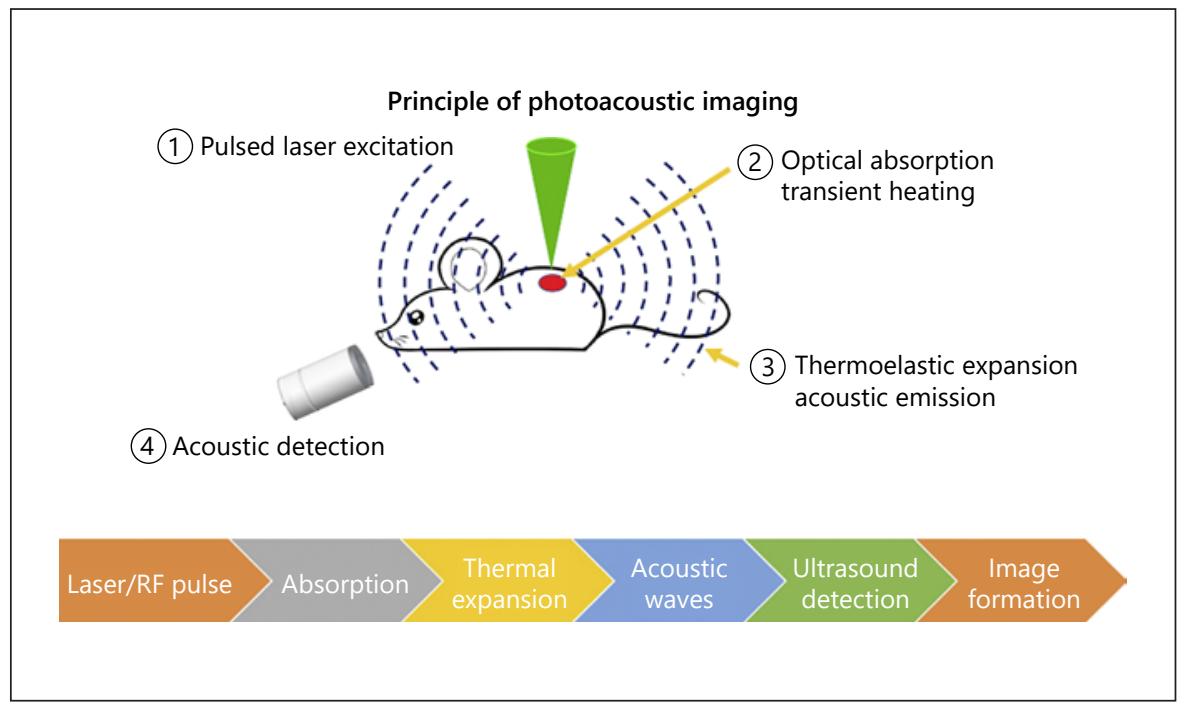

ration of hemoglobin at capillary levels allowing for dynamic monitoring of the metabolic rate of oxygen. This technique has been established and validated for in vivo imaging in the study of brain vasculature [35]. A penetration depth of $200 \mu \mathrm{m}$ allows measurement of hemodynamic changes in peritubular capillaries in the cortex of exteriorized kidneys of an anesthetized mouse. Repetitive measurements can be performed in the same animal over a prolonged period to accurately measure temporal changes after inducing AKI. We are currently employing this method to determine tissue oxygen delivery in the kidney during sepsis. PAM can also be used with other imaging techniques, for example, 2-photon microscopy to simultaneously assess metabolic and microcirculatory changes during heath and disease. Identification of these vital parameters including tissue oxygenation during AKI will permit a better understanding of mechanisms of AKI that will inform therapeutic interventions to improve outcomes of patients with AKI.

\section{Conflict of Interest Statement}

The authors declare no conflict of interest. Research conducted to support data described in this review was supported by the National Science Foundation (2023988) (S.H.). The content is solely the responsibility of the authors and does not necessarily represent the official views of the National Science Foundation.

\section{References}

1 Singer M, Deutschman CS, Seymour CW, Shankar-Hari M, Annane D, Bauer M, et al. The third international consensus definitions for sepsis and septic shock (sepsis-3). JAMA. 2016 Feb 23;315(8):801-10.

2 Bagshaw SM, Lapinsky S, Dial S, Arabi Y, Dodek P, Wood G, et al. Acute kidney injury in septic shock: clinical outcomes and impact of duration of hypotension prior to initiation of antimicrobial therapy. Intensive Care Med. 2009 May;35(5):871-81.

3 Mehta RL, Bouchard J, Soroko SB, Ikizler TA, Paganini EP, Chertow GM, et al. Sepsis as a cause and consequence of acute kidney injury: program to improve care in acute renal disease. Intensive Care Med. 2011 Feb;37(2): 241-8.
4 Vincent JL, Moreno R, Takala J, Willatts S, De Mendonça A, Bruining $\mathrm{H}$, et al. The SOFA (Sepsis-related Organ Failure Assessment) score to describe organ dysfunction/failure. On behalf of the working group on sepsis-related problems of the European society of intensive care medicine. Intensive Care Med. 1996 Jul;22(7):707-10.

5 Gyawali B, Ramakrishna K, Dhamoon AS, Sepsis: the evolution in definition, pathophysiology, and management. SAGE Open Med. 2019;7:2050312119835043.

6 De Backer D, Donadello K, Sakr Y, OspinaTascon G, Salgado D, Scolletta S, et al. Microcirculatory alterations in patients with severe sepsis: impact of time of assessment and relationship with outcome. Crit Care Med. 2013 Mar;41(3):791-9.
7 Poston JT, Koyner JL. Sepsis associated acute kidney injury. BMJ. 2019 Jan 9;364:k4891.

8 Bartz RR, Fu P, Suliman HB, Crowley SD, MacGarvey NC, Welty-Wolf K, et al. Staphylococcus aureus sepsis induces early renal mitochondrial DNA repair and mitochondrial biogenesis in mice. PLoS One. 2014;9(7): e100912.

9 Parikh SM, Yang Y, He L, Tang C, Zhan M, Dong Z. Mitochondrial function and disturbances in the septic kidney. Semin Nephrol. 2015 Jan;35(1):108-19.

10 Molema G, Aird WC. Vascular heterogeneity in the kidney. Semin Nephrol. 2012 Mar; 32(2):145-55. 
11 Eppel GA, Malpas SC, Denton KM, Evans RG. Neural control of renal medullary perfusion. Clin Exp Pharmacol Physiol. 2004 MayJun;31(5-6):387-96.

12 Zhang JL, Morrell G, Rusinek H, Warner L, Vivier PH, Cheung AK, et al. Measurement of renal tissue oxygenation with blood oxygen level-dependent MRI and oxygen transit modeling. Am J Physiol Renal Physiol. 2014 Mar 15;306(6):F579-87.

13 Mathisen O, Monclair T, Kiil F. Oxygen requirement of bicarbonate-dependent sodium reabsorption in the dog kidney. Am J Physiol. 1980 Mar;238(3):F175-80.

14 Aubert V, Kaminski J, Guillaud F, Hauet T, Hannaert P. A computer model of oxygen dynamics in the cortex of the rat kidney at the cell-tissue level. Int J Mol Sci. 2019 Dec 11; 20(24):6246.

15 Simon N, Hertig A. Alteration of fatty acid oxidation in tubular epithelial cells: from acute kidney injury to renal fibrogenesis. Front Med. 2015;2:52.

16 Haase VH. Mechanisms of hypoxia responses in renal tissue. J Am Soc Nephrol. 2013 Mar; 24(4):537-41.

17 Evans RG, Gardiner BS, Smith DW, O'Connor $\mathrm{PM}$. Intrarenal oxygenation: unique challenges and the biophysical basis of homeostasis. Am J Physiol Renal Physiol. 2008 Nov;295(5): F1259-70.

18 De Backer D, Orbegozo Cortes D, Donadello K, Vincent JL. Pathophysiology of microcirculatory dysfunction and the pathogenesis of septic shock. Virulence. 2014 Jan 1;5(1):73-9.

19 Ma S, Evans RG, Iguchi N, Tare M, Parkington HC, Bellomo R, et al. Sepsis-induced acute kidney injury: a disease of the microcirculation. Microcirculation. 2019 Feb;26(2): e12483.
20 Prowle JR, Molan MP, Hornsey E, Bellomo R. Measurement of renal blood flow by phasecontrast magnetic resonance imaging during septic acute kidney injury: a pilot investigation. Crit Care Med. 2012 Jun;40(6):1768-76.

21 Skytte Larsson J, Krumbholz V, Enskog A, Bragadottir G, Redfors B, Ricksten SE. Renal blood flow, glomerular filtration rate, and renal oxygenation in early clinical septic shock. Crit Care Med. 2018 Jun;46(6):e560-e66.

22 Tran M, Tam D, Bardia A, Bhasin M, Rowe GC, Kher A, et al. PGC-1 a promotes recovery after acute kidney injury during systemic inflammation in mice. J Clin Invest. 2011 Oct; 121(10):4003-14.

23 Wang Z, Holthoff JH, Seely KA, Pathak E, Spencer HJ 3rd, Gokden N, et al. Development of oxidative stress in the peritubular capillary microenvironment mediates sepsisinduced renal microcirculatory failure and acute kidney injury. Am J Pathol. 2012 Feb; 180(2):505-16.

24 Vander Heiden MG, Cantley LC, Thompson CB. Understanding the Warburg effect: the metabolic requirements of cell proliferation. Science. 2009 May 22;324(5930):1029-33

25 Gómez H, Kellum JA, Ronco C. Metabolic reprogramming and tolerance during sepsisinduced AKI. Nat Rev Nephrol. 2017 Mar; 13(3):143-51

26 Smith JA, Stallons LJ, Schnellmann RG. Renal cortical hexokinase and pentose phosphate pathway activation through the EGFR/Akt signaling pathway in endotoxin-induced acute kidney injury. Am J Physiol Renal Physiol. 2014 Aug 15;307(4):F435-44.
27 Jin K, Li H, Volpe J, Emlet D, Pastor-Soler N, Pinsky $\mathrm{M}$, et al. Is acute kidney injury in the early phase of sepsis a sign of metabolic downregulation in tubular epithelial cells? Critical Care. 2015;19(Suppl 1):P286.

$28 \mathrm{Li} \mathrm{G}$, Qin Y. Mitochondrial translation factor EF4 regulates oxidative phosphorylation complexes and the production of ROS. Free Radic Res. 2018 Dec;52(11-12):1250-5.

29 Jin L, Zhou Y. Crucial role of the pentose phosphate pathway in malignant tumors. Oncol Lett. 2019 May;17(5):4213-21.

30 Yang L, Xie M, Yang M, Yu Y, Zhu S, Hou W, et al. PKM2 regulates the Warburg effect and promotes HMGB1 release in sepsis. Nat Commun. 2014 Jul 14;5:4436.

31 Baumgärtl H, Zimelka W, Lübbers DW. Evaluation of $\mathrm{PO} 2$ profiles to describe the oxygen pressure field within the tissue. Comp Biochem Physiol A Mol Integr Physiol. 2002 May;132(1):75-85.

32 Kodama Y, Hyodo F, Yamato M, Yasukawa K, Minami Y, Sonoda N, et al. Dynamic nuclear polarization magnetic resonance imaging and the oxygen-sensitive paramagnetic agent OX63 provide a noninvasive quantitative evaluation of kidney hypoxia in diabetic mice. Kidney Int. 2019 Sep;96(3):787-92.

33 Hirakawa Y, Mizukami K, Yoshihara T, Takahashi I, Khulan P, Honda T, et al. Intravital phosphorescence lifetime imaging of the renal cortex accurately measures renal hypoxia. Kidney Int. 2018 Jun;93(6):1483-9.

34 Cao R, Kilroy JP, Ning B, Wang T, Hossack JA, Hu S. Multispectral photoacoustic microscopy based on an optical-acoustic objective. Photoacoustics. 2015 Jun;3(2):55-9.

35 Cao R, Li J, Zhang C, Zuo Z, Hu S. Photoacoustic microscopy of obesity-induced cerebrovascular alterations. Neuroimage. 2019 Mar;188:369-79. 